\title{
Obstructing Gangliocytic Paraganglioma in the Third Portion of the Duodenum
}

\author{
Carlos M. Nuño-Guzmán ${ }^{a} \quad$ José Arróniz-Jáuregui ${ }^{a}$ \\ Francisco Álvarez-López ${ }^{b}$ Jorge L. Coronac \\ Felipe Cerda-Camacho $^{d}$ Rodrigo Rostro $^{a}$ \\ Juan I. Gutiérrez-Manjarrez ${ }^{\mathrm{b}}$
}

Departments of ${ }^{\mathrm{a}}$ General Surgery, ${ }^{\mathrm{b}}$ Gastroenterology, ${ }^{\mathrm{c}}$ Radiology and

${ }^{d}$ Pathologic Anatomy, Antiguo Hospital Civil de Guadalajara 'Fray Antonio

Alcalde', Guadalajara, Mexico

\section{Key Words}

Duodenal obstruction · Gangliocytic paraganglioma - Duodenal neoplasm

\begin{abstract}
Gangliocytic paragangliomas are infrequent tumors almost exclusively found in the second portion of the duodenum. An unusual case of a gangliocytic paraganglioma in the third portion of the duodenum with obstructive symptoms is herein reported. A 16-year-old male patient presented with epigastric pain, postprandial plenitude and reflux. A barium swallow failed to demonstrate abnormalities. Endoscopy showed a pedunculated submucosal tumor, originating at the third duodenal portion and causing partial obstruction. Biopsy was not performed due to the risk of bleeding. CT scan demonstrated a polypoid lesion. Through a transmesocolic approach and an anterior duodenotomy, resection of the tumor was performed. No lymph node or other organ affection was found. Histologic examination revealed a gangliocytic paraganglioma. Immunohistochemical examination was performed. Gangliocytic paragangliomas originating in the third or fourth portion of the duodenum, as in the present case, are extremely rare. Characteristic histologic features including epithelioid cells, spindle-shaped cells and ganglion-like cells were met. The majority of cases manifest with a similar benign behavior. Local resection of the tumor is recommended for these cases. An infrequent case of a gangliocytic paraganglioma located in the third portion of the duodenum, with a less common clinical presentation, is herein reported.
\end{abstract}




\section{Introduction}

Gangliocytic paragangliomas are infrequent tumors of the gastrointestinal tract usually found in the second portion of the duodenum. The most common clinical presentation includes gastrointestinal bleeding, followed by abdominal pain and anemia. Compromise of the ampulla of Vater has also been described. These submucosal tumors have been generally considered as benign and non-functional. Histologic diagnosis requires the identification of three elements, namely epithelioid cells, spindle-shaped cells and ganglion-like cells $[1,2]$.

Dahl et al. [3] in 1957 reported the first case of gangliocytic paraganglioma. Most of the tumor consisted of ganglion cells of varying shape and size and impregnable nerve fibers. Taylor and Helwig [4] in 1962 described a series of polypoid duodenal tumors which they called benign non-chromaffin paragangliomas. All were located in the second portion of the duodenum, with predilection for the papilla of Vater. Spindle cell and epithelioid cell elements were observed together with ganglion-like cells. Lukash et al. [5] in 1966 reported a polypoid tumor in the duodenum, histologically identical with the previously described non-chromaffin paragangliomas. This lesion was located in the fourth portion, near the ligament of Treitz. Kepes and Zacharias [6] in 1971 reported two cases of benign polypoid submucosal tumors of the duodenum. The tumors were composed of clusters and ribbons of columnar epithelioid cells with transitions to more spindly elements and to well-developed ganglion cells. They suggested the term 'gangliocytic paraganglioma' for these lesions, which showed microscopic features seen in paragangliomas as well as ganglioneuromas. These tumors are submucosal and non-functional, typically composed of epithelioid cell nests, areas of spindle cells and scattered ganglion cells.

The exact origin of gangliocytic paragangliomas is unknown. The most common clinical manifestation is gastrointestinal bleeding, followed by abdominal pain and anemia. A less common presentation is biliary obstruction secondary to compromise of the papilla of Vater [2]. Rarely, gangliocytic paragangliomas manifest by pyloric or duodenal obstruction. A benign behavior at long-term follow-up (1-25 years, mean 8.3 years) after resection has been reported [7].

The most common location of gangliocytic paragangliomas in the gastrointestinal tract is the duodenum, particularly the second portion [2, 7]. Gangliocytic paragangliomas originating in the third or fourth portion of the duodenum are extremely rare. An unusual case of a gangliocytic paraganglioma originating in the third portion of the duodenum manifesting with obstructive symptoms is herein described.

\section{Case Report}

A 16-year-old male patient presented to our hospital with progressively increasing epigastric pain, postprandial plenitude and reflux, limiting his oral intake. He had experienced weight loss of approximately $10 \mathrm{~kg}$ during the previous 12 months. He had been previously treated with $\mathrm{H} 2$ blockers and proton pump inhibitors without significant relief. There were no data suggesting gastrointestinal bleeding. Laboratory blood tests were unremarkable. Upper gastrointestinal tract evaluation with barium swallow failed to demonstrate any abnormality. Upper gastrointestinal endoscopy showed mild esophagitis and mild edematous gastritis; the first and second duodenal portions appeared normal, whereas further examination showed a $2.5 \times 2 \mathrm{~cm}$ pedunculated submucosal tumor partially 
obstructing the duodenum at the third and fourth portion. Biopsy was not performed due to the risk of bleeding. A CT scan was performed and showed an endoluminal polypoid lesion originating in the third duodenal portion, protruding into the proximal jejunum (fig. 1). The patient was scheduled for surgery. Due to the location of the tumor, a transmesocolic approach was performed, with dissection at the duodenojejunal junction. Proximal duodenal dissection was carried out until the pedicle base was easily handled (fig. 2a). Through an anterior duodenotomy, resection at the base of the pedicle was performed. The mucosa layer was repaired with absorbable suture. No lymph node or other organ affection was found. After an uneventful recovery and oral intake on the third postoperative day, the patient was discharged.

Upon macroscopic examination, a $2.3 \times 1.9 \times 2 \mathrm{~cm}$ submucous polypoid, non-encapsulated and well-delimited tumor of moderately firm consistency, covered by normal aspect mucosa, was observed (fig. 2b). The $0.9 \mathrm{~cm}$ implantation base showed no infiltration of adjacent tissue, and a $1.7 \mathrm{~cm}$ extension and $0.4 \mathrm{~cm}$ thick round border of healthy tissue was found. On histologic examination, a solid and trabecular pattern neoplasia was observed (fig. $3 \mathrm{a}, \mathrm{b}$ ), with various cellular subtypes: polygonal and compact nests of epithelioid cells simulating a carcinoid tumor, spindle-shaped cells with a neurofibrillar aspect cytoplasm, and large cells with gangliocytic differentiation, diagnostic of a gangliocytic paraganglioma (fig. 3c-e). The lesion was confined to the submucosa, with no evidence of lymphovascular invasion and no mitotic activity. The overlying mucosa showed reactive and inflammatory changes only. The resection margins were tumor-free. On immunohistochemical examination, positive stain for pancreatic polypeptide, neuron-specific enolase, protein S-100 and chromogranin-A was observed (fig. $3 \mathrm{f}-\mathrm{i}$ ). Positive stain for synaptophysin, progesterone receptors and Ki-67 was observed as well. Stain for estrogen receptors, CD117 (c-kit) and p53 was negative (fig. 3j-l). Fifteen months after the surgery the patient remains free of symptoms, has recovered weight and continues follow-up.

\section{Discussion}

Gangliocytic paragangliomas are infrequent submucosal tumors of the gastrointestinal tract usually found in the second portion of the duodenum [1, 2]. Several theories about the origin of gangliocytic paragangliomas have been proposed: origin from an embryonic celiac ganglion; the tumor deriving from pluripotent stem cells located at the base of intestinal glands; the lesion deriving from hamartomatous proliferation of ectodermally derived epithelial cells originating from the ventral primordium of the pancreas, and neuroectodermally derived ganglion and schwannian spindle cell; and the lesion arising from ectopic pancreatic tissue $[1,4,8]$.

The mean age at presentation is 52 years (range 15-84), with a male predominance (1.5:1). The most common clinical manifestation is gastrointestinal bleeding (45.1\%) due to mucosal erosion or ulceration, followed by abdominal pain $(42.8 \%)$ and anemia $(14.5 \%)$. A less common presentation is biliary obstruction secondary to compromise of the papilla of Vater (4.6\%) [2]. Some cases have been incidentally found at autopsy. Rarely, gangliocytic paragangliomas manifest by pyloric or duodenal obstruction due to a huge mass effect. The most common location of gangliocytic paragangliomas in the gastrointestinal tract is the duodenum (90.1\%), while less common sites are the jejunum, stomach, appendix and pancreas. The vast majority of duodenal gangliocytic paragangliomas are located in the second portion [2, 7]. Exceptional cases of gangliocytic paragangliomas originating in the third or fourth portion of the duodenum have been reported [5, 7, 9-12].

Adequate endoscopic biopsy for a precise histologic identification may be difficult due to the submucosal nature of gangliocytic paragangliomas. The diagnostic rate by endoscopic biopsy has been $11.4 \%$ only [2]. Imaging features are helpful in 
diagnostic determination. Barium swallow may show a polypoid, soft-surfaced, intraluminal mass effect; in our case this feature was not observed. Contrast-enhanced TC scan will usually demonstrate a pedunculated or intramural mass with soft tissue attenuation and homogeneous enhancement. On magnetic resonance imaging, gadolinium-enhanced images show homogeneous enhancement of the lesion. The solid appearance and homogeneous contrast enhancement features are helpful in determining the possible diagnosis. Common bile duct dilatation depends on tumor location and compromise of the papilla of Vater [13].

Endoscopic ultrasound features of gangliocytic paragangliomas are isoechoic or hypoechoic, heterogeneous tumor with smooth margins, originating from the submucosal layer of the duodenum. The tumor is usually polypoid, but may be sessile or pedunculated. Lymphadenopathy can also be assessed. Endoscopic ultrasound is not useful for the precise diagnosis of this tumor due to the absence of a pathognomonic pattern. However, it provides accurate information about the local and regional staging of the tumor $[8,11]$.

Differential diagnosis includes pancreatic head or duodenal cancer, choledochal cyst, duodenal tumors such as lipoma, hamartoma, hemangioma, lymphoma, leiomyosarcoma and inflammatory fibroid polyps. This differentiation can be performed radiologically, based on tumor location, degree of attenuation by abdominal CT, common bile duct dilatation and enhancing pattern [13].

The characteristic histologic features in gangliocytic paragangliomas are epithelioid cells with an endocrine growth pattern, spindle-shaped cells and ganglion-like cells. The lesion is submucosal, non-encapsulated and well-circumscribed $[1,6]$. Immunohistochemical analysis may show reactivity for neuron-specific enolase, synaptophysin, pancreatic polypeptide, somatostatin, chromogranin-A, cytokeratin, serotonin, myelin basic protein, neurofilament protein, S-100 protein and neuron-specific enolase [2].

Most gangliocytic paragangliomas are benign and can be treated by local resection [7]. When considered possible and safe, the treatment of choice for gangliocytic paragangliomas is endoscopic resection. Tumor location, depth of invasion, risks of procedure-related complications and possible lymph node affection should be considered. When the endoscopic approach is not considered possible or safe, surgical options include local resection or ampullectomy for those tumors affecting the papilla of Vater. Although the majority of gangliocytic paragangliomas manifest with a benign behavior, metastatic affection of regional lymph nodes and distant organs has been reported, particularly in ampullary gangliocytic paragangliomas [14]. Moreover, there is a high rate of gangliocytic paragangliomas exceeding the submucosal layer, a risk factor for lymph node metastasis, which makes CT particularly important [2]. An aggressive behavior may also be suggested by the presence of nuclear pleomorphism, mitotic activity and infiltrative margin [15]. In the case of ampullary paragangliomas, these findings favor radical surgical resection [14]. Gangliocytic paragangliomas of the third or fourth portion of the duodenum are extremely rare, which limits the possibility of consensus for management. Clinical manifestations may be due to obstruction or bleeding [5, 7, 9-12]. A clinical, tomographic and endoscopic evaluation is recommended, as well as surgical exploration and histologic confirmation of the resected tumor. 
In the present case of gangliocytic paraganglioma, symptoms were of obstructive nature, while there were no data of gastrointestinal bleeding, the most common presentation. CT showed a polypoid tumor originating in the third duodenal portion. A proximal endoscopy identified the submucosal tumor, partially obstructing the duodenum at the third and fourth portion. Local surgical resection was performed. The tumor was confined to the submucosa, and no evidence of malignancy was found. In conclusion, a rare case of a gangliocytic paraganglioma originating in the third portion of the duodenum is herein reported. Less common clinical manifestations characterized by obstructive symptoms were present.

\section{Disclosure Statement}

The authors have no conflicts of interest, sponsorship or funding arrangements to declare.

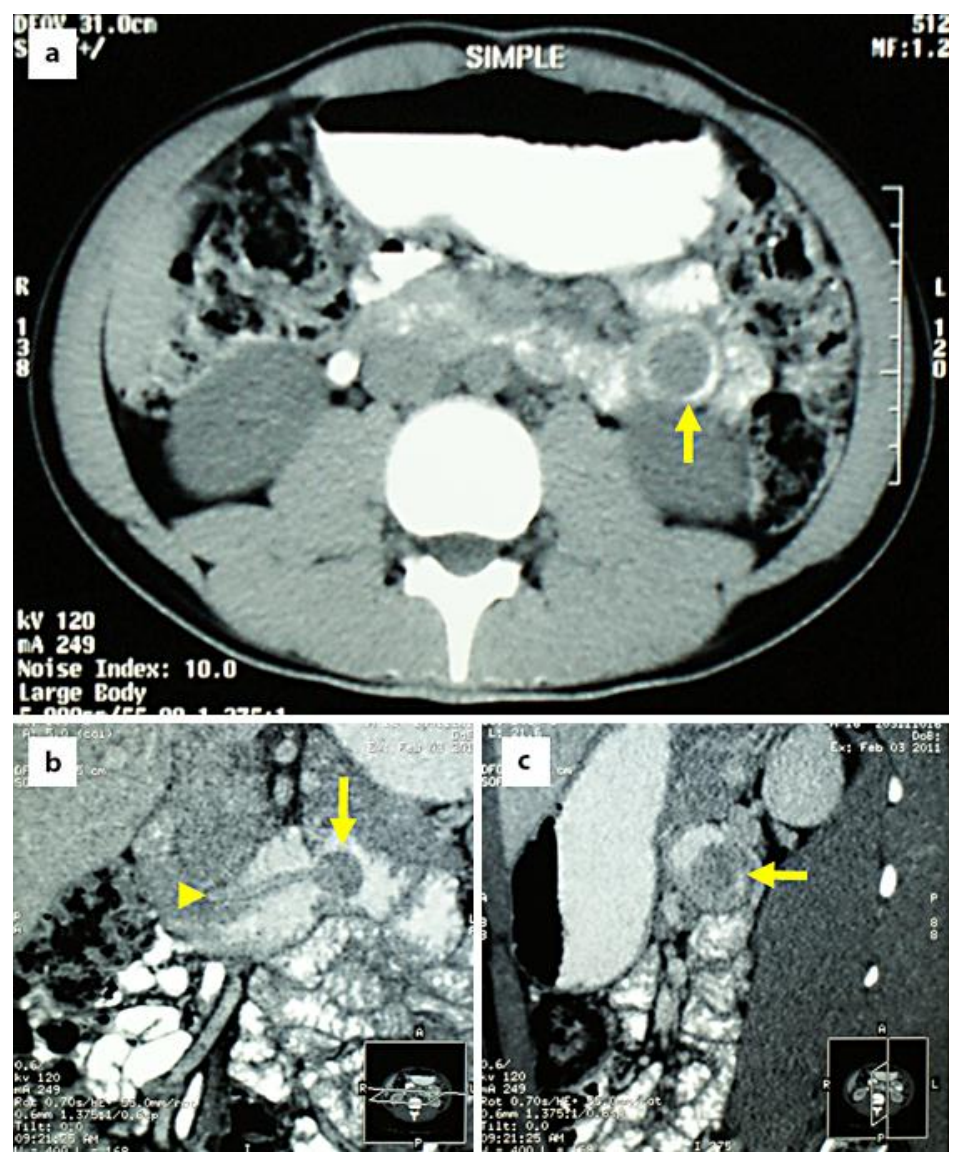

Fig. 1. a CT scan demonstrated an endoluminal polypoid tumor protruding into the proximal jejunum (arrow). b Coronal view demonstrated a pedunculated polyp (arrow) with a $2.5 \mathrm{~cm}$ pedicle originating at the third portion of duodenum (arrowhead). c Sagittal view demonstrates a polyp in the third duodenal portion (arrow). 


\begin{tabular}{r|l|l|l}
$\begin{array}{r}\text { Case Reports in } \\
\text { Gastroenterology }\end{array}$ & $\begin{array}{l}\text { Case Rep Gastroenterol 2012;6:489-495 } \\
\text { DOI: 10.1159/000341586 }\end{array}$ & $\begin{array}{l}\text { Published online: } \\
\text { July 24, 2012 }\end{array}$ & $\begin{array}{l}\text { @ 2012 S. Karger AG, Basel } \\
\text { ISSN 1662-0631 } \\
\text { www.karger.com/crg }\end{array}$ \\
\hline
\end{tabular}

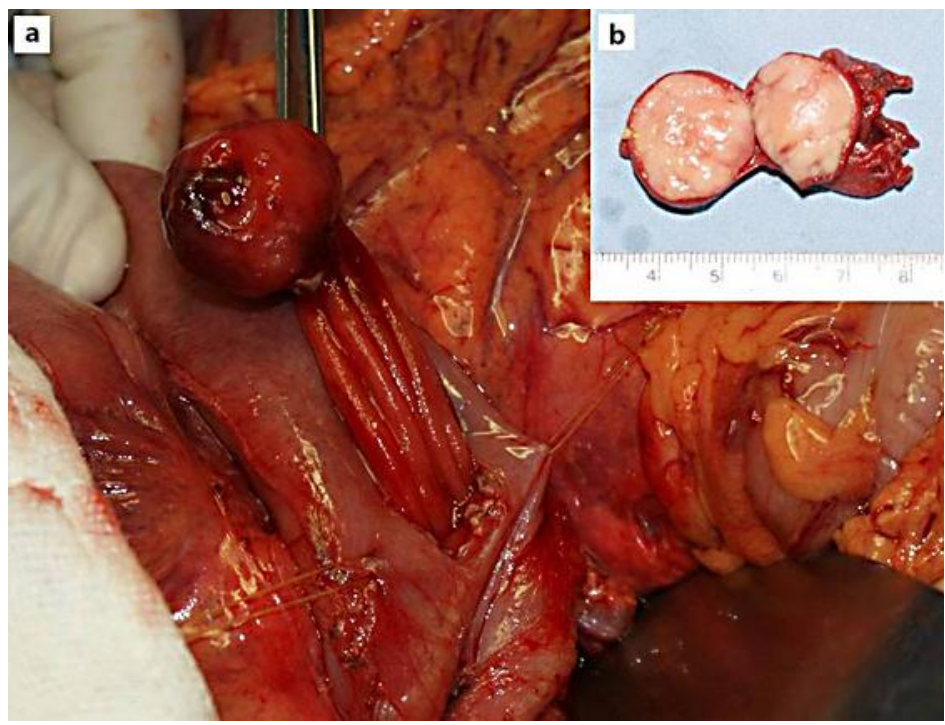

Fig. 2. a The surgical aspect showed, through a transmesocolic approach and an anterior duodenotomy, the pedunculated polypoid lesion under traction. $\mathbf{b}$ The macroscopic aspect showed a $2.3 \times 1.9 \times 2 \mathrm{~cm}$, submucous and well-delimited polypoid tumor. The lesion was moderately firm and covered with normal aspect duodenal mucosa.

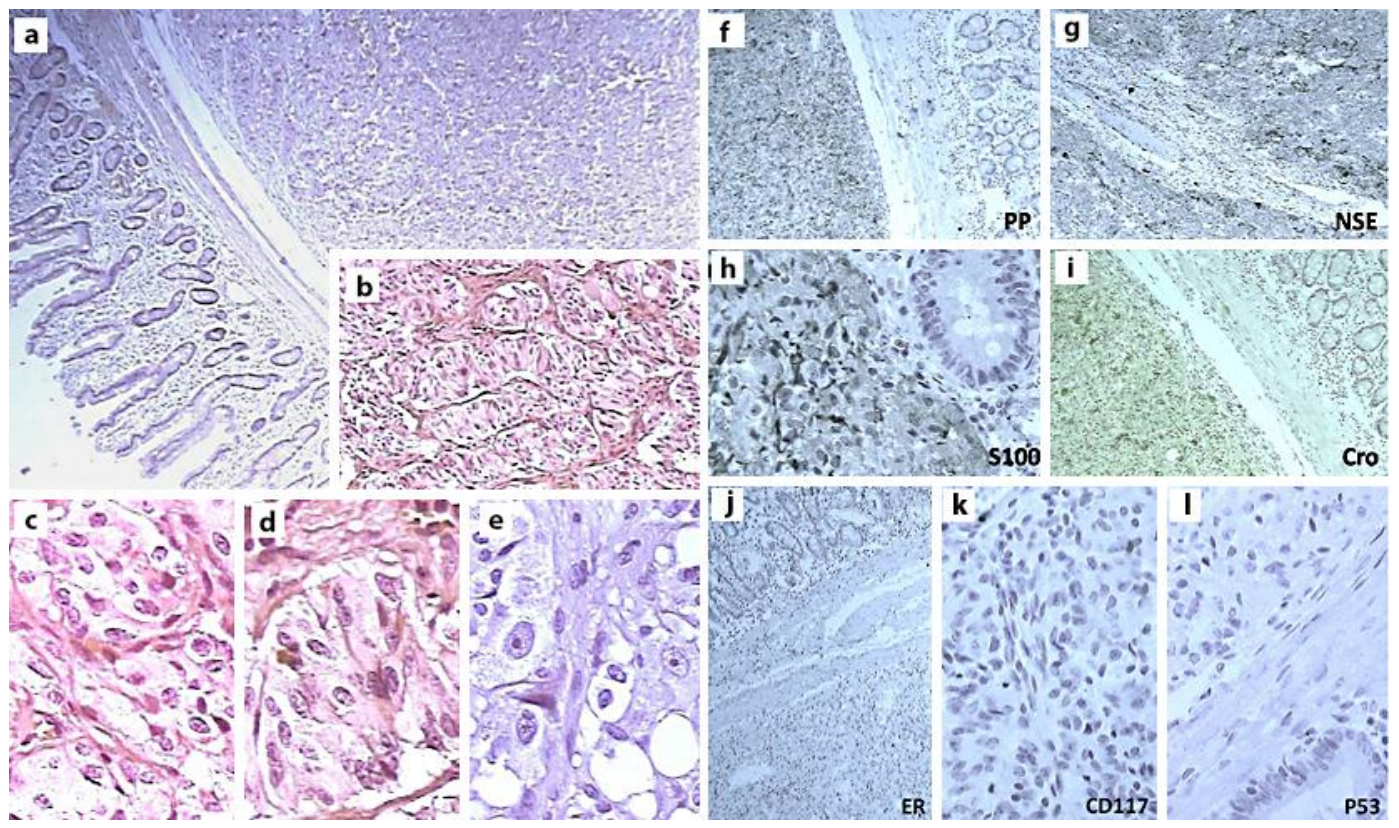

Fig. 3. Histologic aspect which demonstrates a solid, submucous tumor (H\&E, 100x) (a) and trabecular pattern (H\&E, 400x) (b). Cellular components: c polygonal epithelioid cells; d spindle-shaped cells; e ganglion-like cells (H\&E, 400×). Immunohistochemical analysis revealed positive reactivity for pancreatic polypeptide (f), neuron-specific enolase (g), protein S-100 (h) and chromogranin-A (i). Negative reactivity for estrogen receptors (j), CD117 (k) and p53 (I) was revealed. 


\section{References}

1 Burke AP, Helwig EB: Gangliocytic paraganglioma. Am J Clin Pathol 1989;92:1-9.

2 Okubo Y, Wakayama M, Nemoto T, Kitahara K, Nakayama H, Shibuya K, Yokose T, Yamada M, Shimodaira K, Sasai D, Ishiwatari T, Tsuchiya M, Hiruta N: Literature survey on epidemiology and pathology of gangliocytic paraganglioma. BMC Cancer 2011;11:187.

3 Dahl EV, Waugh JM, Dahlin DC: Gastrointestinal ganglioneuromas; brief review with report of a duodenal ganglioneuroma. Am J Pathol 1957;33:953-965.

-4 Taylor HB, Helwig EB: Benign nonchromaffin paragangliomas of the duodenum. Virchows Arch Pathol Anat Physiol Klin Med 1962;335:356-366.

5 Lukash WM, Hyams VJ, Nielsen OF: Neurogenic neoplasms of the small bowel: benign nonchromaffin paraganglioma of the duodenum. Am J Dig Dis 1966;11:575-579.

-6 Kepes JJ, Zacharias DL: Gangliocytic paragangliomas of the duodenum. A report of two cases with light and electron microscopic examination. Cancer 1971;27:61-67.

-7 Scheithauer BW, Nora FE, LeChago J, Wick MR, Crawford BG, Weiland LH, Carney JA: Duodenal gangliocytic paraganglioma. Clinicopathologic and immunocytochemical study of 11 cases. Am J Clin Pathol 1986;86:559-565.

8 Perrone T, Sibley RK, Rosai J: Duodenal gangliocytic paraganglioma: an immunohistochemical and ultrastructural study and a hypothesis concerning its origin. Am J Surg Pathol 1985;9:31-41.

-9 Smithline AE, Hawes RH, Kopecky KK, Cummings OW, Kumar S: Gangliocytic paraganglioma, a rare cause of upper gastrointestinal bleeding. Endoscopic ultrasound findings presented. Dig Dis Sci 1993;38: 173-177.

10 Nagai T, Torishima R, Nakashima H, Tanahashi J, Iwata M, Ookawara H, Yokoyama S, Yada K, Sato R, Murakami K, Fujioka T: Duodenal gangliocytic paraganglioma treated with endoscopic hemostasis and resection. J Gastroenterol 2004;39:277-283.

11 Mann CM, Bramhall SR, Buckels JA, Taniere P: An unusual case of duodenal obstruction-gangliocytic paraganglioma. J Hepatobiliary Pancreat Surg 2009;16:562-565.

12 Sucandy I, Ayers G, Bertsch DJ: Surgical management of endoscopically unresectable duodenal gangliocytic paraganglioma in a patient with partial upper gastrointestinal obstruction. $\mathrm{N} \mathrm{Am} \mathrm{J} \mathrm{Med} \mathrm{Sci}$ 2010;2:547-551.

13 Buetow PC, Levine MS, Buck JL, Pantongrag-Brown L, Emory TS: Duodenal gangliocytic paraganglioma: CT, MR imaging and US findings. Radiology 1997;204:745-747.

14 Ghassemi KA, Cortina G, Reber HA, Farrell JJ: Complete resection of ampullary paragangliomas confined to the submucosa on endoscopic ultrasound may be best achieved by radical surgical resection. Case Rep Gastroenterol 2009;3:169-174.

15 Witkiewics A, Galler A, Yeo CJ, Gross SD: Gangliocytic paraganglioma: case report and review of the literature. J Gastrointest Surg 2007;11:1351-1354. 